\title{
Short-Term Effects of Vagus Nerve Stimulation on Learning and Evoked Activity in Auditory Cortex
}

\author{
Jesyin Lai and ${ }^{-S t e p h e n ~ V . ~ D a v i d ~}$ \\ https://doi.org/10.1523/ENEURO.0522-20.2021 \\ Oregon Research Hearing Center, Oregon Health and Science University, Portland, Oregon
}

\begin{abstract}
Chronic vagus nerve stimulation (VNS) has been shown to facilitate learning, but effects of acute VNS on neural coding and behavior remain less well understood. Ferrets implanted with cuff electrodes on the vagus nerve were trained by classical conditioning on an auditory tone frequency-reward association. One tone was associated with reward while another tone was not. Tone frequencies and reward associations were changed every $2 \mathrm{~d}$, requiring learning of a new relationship. When tones were paired with VNS, animals consistently learned the new association within $2 \mathrm{~d}$. When VNS occurred randomly between trials, learning within $2 \mathrm{~d}$ was unreliable. In passively listening animals, neural activity in primary auditory cortex (A1) and pupil size were recorded before and after acute VNS-tone pairing. After pairing with a neuron's best-frequency (BF) tone, responses by a subpopulation of neurons were reduced. VNS paired with an off-BF tone or during intertrial intervals had no effect. The BF-specific reduction in neural responses after VNS remained, even after regressing out changes explained by pupil-indexed arousal. VNS induced brief dilation in the pupil, and the size of this change predicted the magnitude of persistent changes in the neural response. This interaction suggests that fluctuations in neuromodulation associated with arousal gate the long-term VNS effects on neural activity.
\end{abstract}

Key words: auditory; vagus nerve; plasticity; reward; learning; pupil

\section{Significance Statement}

Vagus nerve stimulation (VNS) has been demonstrated to facilitate learning of sensory and motor behaviors. It is believed to trigger neuromodulator release that mediates cortical plasticity associated with learning. This study explores short-term VNS effects that can support long-term plasticity in the auditory cortex (A1). Just $2 \mathrm{~d}$ of VNS were adequate to support enhanced learning of an auditory discrimination task. Neural recordings from A1 revealed briefly pairing VNS with a neuron's best-frequency (BF) tone reduced responses in a subpopulation of neurons. This reduction persisted even after regressing out responses explained by pupil size, a measure of global arousal. These results support a role for VNS in auditory learning and help establish VNS as a tool to facilitate neural plasticity.

\section{Introduction}

Chronic vagus nerve stimulation (VNS) has been reported to improve learning and memory in humans (Clark et al., 1999) and rats (Clark et al., 1995). Previous studies have demonstrated that VNS during rehabilitative training improves recovery of motor function in several models of brain injury (Hays et al., 2014a,b, 2016; Khodaparast et al., 2014, 2016; Meyers et al., 2018). The therapeutic

Received December 1, 2020; accepted April 16, 2021; First published June 2, 2021.

The authors declare no competing financial interests. benefits of VNS during motor rehabilitation persist even after the cessation of stimulation, suggesting that VNS-induced plasticity and learning are long-term (Hays et al., 2014a; Khodaparast et al., 2016). VNS has been used in clinical therapies for epilepsy, depression and other neurologic disorders (Groves and Brown, 2005). VNS has also been reported to enhance memory and to facilitate extinction of fear conditioning in rats (Peña et al., 2013, 2014). These diverse findings suggest that VNS facilitates wide-

Author contributions: J.L. and S.V.D. designed research; J.L. performed research; J.L. analyzed data; J.L. and S.V.D. wrote the paper. 
ranging neural plasticity, which could also support learning of new auditory categories.

Although VNS has multiple clinical applications, studies investigating mechanisms by which VNS facilitates neural plasticity are limited. Recruitment of neuromodulatory activity by VNS is believed to contribute to enhanced learning (McGaugh, 1989; Engineer et al., 2015). Both the cholinergic and adrenergic systems have been implicated as playing a role. Afferent signals from VNS have been reported to activate widespread release of these neuromodulators in the brain via the nucleus tractus solitarius, the locus coeruleus (LC) and nucleus basalis (NB; Dorr and Debonnel, 2006; Engineer et al., 2013).

Most previous studies of VNS-mediated plasticity in the auditory system have measured plasticity following chronic VNS, e.g., 300 times/d for $20 \mathrm{~d}$ (Engineer et al., 2011, 2015, 2017; Shetake et al., 2012; Borland et al., 2019). Here, short-term effects of VNS on auditory learning and cortical plasticity were explored. To study behavioral effects of VNS, a paradigm was developed to measure reward association learning over 200-500 trials (1-2d). Ferrets were trained by classical conditioning to discriminate between rewarded (conditioned stimulus positive; CS+) and non-rewarded (CS-) tones, and the rate of learning was compared with and without VNS. To measure effects of VNS on cortical activity, single-unit and multiunit neural activity was recorded in primary auditory cortex (A1) of passively listening animals. In subsequent neurophysiological recordings, VNS was paired with tone stimuli similar to those used for behavior. To determine the interaction between VNS, arousal and neurophysiological activity, pupil size, a measure of global arousal, was recorded during the passive stimulation experiments. Linear regression was used to dissociate effects of pupil-indexed arousal from A1 plasticity following VNS.

\section{Materials and Methods}

\section{Ethics statement}

All procedures were performed in accordance with the Oregon Health and Science University Institutional Animal Care and Use Committee and conform to standards of the Association for Assessment and Accreditation of Laboratory Animal Care (AAALAC).

This work was supported by Defense Advanced Research Projects Agency Grant D15AP00101 and the National Institute on Deafness and Other Communication Disorders Grant R01 DC014950.

Acknowledgements: We thank Luke A. Shaheen in assistance with cuff electrode testing and data analysis of array recording; Luke A. Shaheen, Zachary P. Schwartz, and Daniela Saderi for assistance with behavioral training and neurophysiological recording; and Zachary P. Schwartz and Charles R. Heller for technical assistance with pupillometry.

Correspondence should be addressed to Stephen V. David at davids@ ohsu.edu.

https://doi.org/10.1523/ENEURO.0522-20.2021

Copyright (C) 2021 Lai and David

This is an open-access article distributed under the terms of the Creative Commons Attribution 4.0 International license, which permits unrestricted use, distribution and reproduction in any medium provided that the original work is properly attributed.

\section{Animals}

Three young adult male ferrets (animals $\mathrm{P}, \mathrm{S}$, and $\mathrm{N}$, less than nine months) were obtained from an animal supplier (Marshall Farms). Ferrets were used in this study because they have broad hearing frequency range that overlaps with that of humans. Moreover, ferrets are relatively easy to train and have an established repertoire of auditory behaviors (Fritz et al., 2003; David et al., 2012). The zeitgeber time (ZT) of the animal facility was $Z T 0=6$ A.M. and $Z \mathrm{TT} 12=6$ P.M. Before experiments, animals were implanted with a steel post for head fixation and to expose a portion of the skull for access to auditory cortex. Anesthesia was induced using ketamine $(35 \mathrm{mg} / \mathrm{kg}$, i.m.) and xylazine $(5 \mathrm{mg} / \mathrm{kg}$, i.m.) and maintained with isoflurane $(0.5-2 \%)$. A warmed saline solution $(10 \mathrm{ml})$ was given to the animals to prevent dehydration. Anesthesia depth was monitored by heart rate, respiration rate and blood oxygen percentage. Under sterile conditions, the headpost was mounted to the skull using dental acrylic (AM Systems) or Charisma composite, which bonded to the skull and to a set of stainless-steel screws embedded in the bone. After the surgery, animals were treated with prophylactic antibiotics (Baytril, $100 \mathrm{mg} / \mathrm{ml}$, s.c.) and analgesics (buprenorphine, $0.02 \mathrm{mg} / \mathrm{kg}$, s.c.) under the supervision of the university veterinary staff. The wound was cleaned and bandaged during a two-week recovery period. After recovery, each ferret was gradually acclimated to head fixation using a custom stereotaxic apparatus in a Plexiglas tube. Habituation sessions initially lasted for $5 \mathrm{~min}$ and increased by increments of 5-10 min daily until the ferret rested comfortably in the tube for at least $1 \mathrm{~h}$. Timelines summarizing the sequence of surgeries, behavioral training, and neurophysiological recordings are reported in Figure 1B,C.

\section{Vagus nerve implant surgery}

After acclimation to head fixation, each animal was implanted with a tripolar cuff electrode (Cortec or Microprobes) around the left cervical vagus nerve (Engineer et al., 2011), using a protocol adapted from work in rats (Lu et al., 2018). Since the right vagus nerve innervates the sinoatrial node, VNS on the right nerve may negatively impact heart rate (Johnson and Wilson, 2018). As a result, we chose to perform VNS on the left vagus nerve. Induction and other aspects of the surgery were the same as for the headpost implant.

Animals were placed in a supine position for implantation of the electrode cuff. Lidocaine ( $2 \mathrm{mg} / \mathrm{kg}$, s.c.) was injected in the neck at the incision site, and the left cervical vagus nerve was exposed through blunt dissection of the neck and detachment from the carotid artery. The cuff was secured around the nerve, and leads from the electrode were tunneled subcutaneously to the headpost. They were then secured to the headpost implant with acrylic.

Efficacy of VNS was confirmed by observation of a heart rate drop while stimulating the nerve through the electrode leads (AM Systems 2100). Upon confirmation that the cuff was providing stimulation, the neck was sutured closed and Bacitracin antibiotic cream was applied to incision sites on the neck and head. Animals were 
A

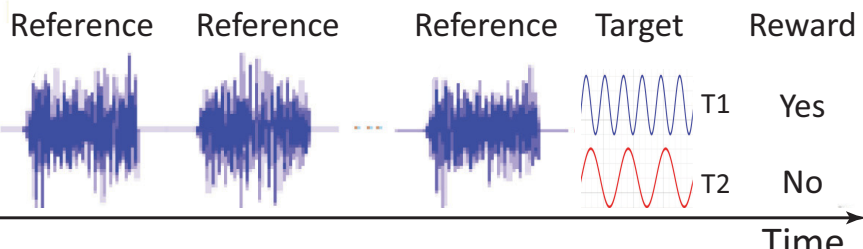

Stimuli

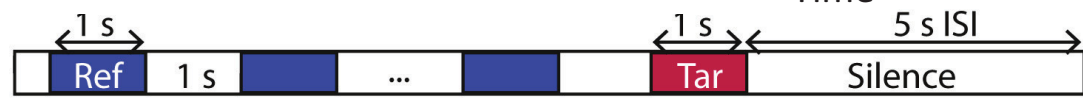

Lick

Resp

Late response

VNS

\section{B}

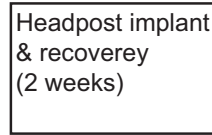

Early response
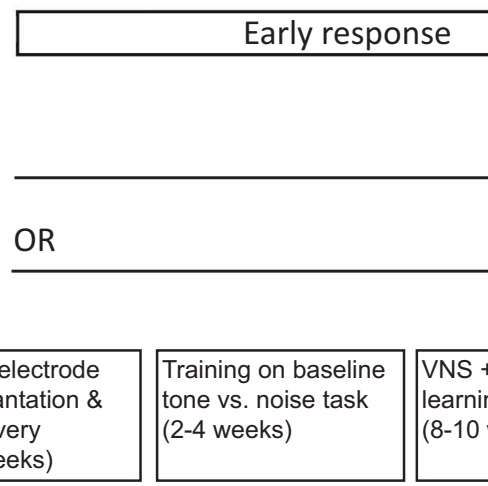

paired

-150 or -100 ms Tar Onset

unpaireld

Random during ISI

C VNS + behavior timeline

\begin{tabular}{|l|c|c|c|c|c|c|c|c|c|}
\hline Training days (200 trials/day) & $1-2$ & $3-4$ & $5-6$ & $7-8$ & $9-10$ & $11-12$ & $13-14$ & $15-16$ & \\
\hline $\begin{array}{l}\text { VNS paired (P) or unpaired (U) } \\
\text { with tones }\end{array}$ & $\mathrm{U}$ & $\mathrm{P}$ & $\mathrm{U}$ & $\mathrm{P}$ & $\mathrm{U}$ & $\mathrm{P}$ & $\mathrm{U}$ & $\mathrm{P}$ & $\ldots$ \\
\hline $\begin{array}{l}\text { Rewarded target (T1) relative } \\
\text { frequency high (H) / low (L) }\end{array}$ & $\mathrm{H}$ & $\mathrm{H}$ & $\mathrm{L}$ & $\mathrm{L}$ & $\mathrm{H}$ & $\mathrm{H}$ & $\mathrm{L}$ & $\mathrm{L}$ & \\
\hline
\end{tabular}

Figure 1. Classical conditioning behavior and experimental timelines. A, Animals were trained using classical conditioning to associate one target tone (T1) with a reward and another target tone (T2) with no reward. T1 and T2 were changed every $2 \mathrm{~d}$ (200-250 trials/d), typically after target-reward associations were learned. During these $2 \mathrm{~d}$ of training, T1 and T2 were either paired with VNS (1-s duration, $30 \mathrm{~Hz}, 200-\mu$ s biphasic pulses, 0.4-2 mA, VNS onset 100 or $150 \mathrm{~ms}$ before T1/T2 onset) or unpaired with VNS (occurring randomly during the interstimulus interval, ISI). One or more licks during target presentation (0.2-1.15 $\mathrm{s}$ after tone onset) were considered a response to the target sound in anticipation of a reward. $\boldsymbol{B}$, Timeline reporting the broad sequence of surgeries, behavioral training, and neurophysiological recordings for each animal. $\boldsymbol{C}$, Table showing an example of behavioral training sessions for tone-reward association tasks across multiple days. The table includes different transitions for paired $(P)$ and unpaired $(U)$ VNS as well as whether T1 was the higher $(\mathrm{H})$ or lower $(\mathrm{L})$ tone. The sequences of these transitions were shuffled among different animals. Animals were trained for several weeks, two to three 2-d blocks per week.

given Baytril (10 mg/kg, s.c.) and buprenorphine $(0.02 \mathrm{mg} /$ $\mathrm{kg}$, s.c.) for $2 \mathrm{~d}$ after surgery.

\section{Validation of cuff electrode function}

To confirm function of the cuff electrode following implantation, effects of VNS on heart rate (under anesthesia) and pupil size (awake, passive condition) were assessed separately and on different days. For heart rate measurement, animals were anesthetized with ketamine $(5 \mathrm{mg} / \mathrm{kg}$, i.m.) and dexmedetomidine ( $0.05 \mathrm{mg} / \mathrm{kg}$, i.m.). Atropine $(0.05 \mathrm{mg} / \mathrm{kg}$, i.m.) was injected to prevent bradycardia. Without atropine, heart rate fluctuation was observed, which sometimes masked VNS effects. Animals' heart rate was compared immediately before and after delivery of current to the cuff (0.1-2 mA, 200- $\mu$ s biphasic pulses at $30 \mathrm{~Hz}, 3-5 \mathrm{~s}$ in duration), and a drop in heart rate following
VNS indicated effective stimulation. For pupil size measurement, animals were awake and head-fixed but not behaving. Pupillometry was obtained by infrared video before and after VNS, as described below.

\section{Pupillometry}

During VNS and neurophysiological recordings, the pupil in one eye was recorded using an open-source video camera (Adafruit TTL Serial Camera) fitted with a macro lens (M12 Lenses PT-2514BMP, 25 mm). The camera was placed $10 \mathrm{~cm}$ from the animal's eye. To improve contrast, the imaged eye was illuminated by a bank of infrared LEDs. Visible light was provided at constant intensity using a ring light (AmScope LED-144S), adjusted so that a maximum dynamic range of pupil size could be measured. 
In early pupillometry datasets, pupil size was measured using custom MATLAB code (Schwartz et al., 2020). For later recordings, a deep neural network (DNN) was trained using Python to locate the pupil in each video frame and fit an ellipse to the pupil boundary. The algorithm was trained on video frames that were labeled using the methods in Schwartz et al. (2020). After training, the DNN performed well on novel video frames from new animals, producing results consistent with the MATLAB method. If the fit quality was poor, additional frames from the dataset were labeled, and the model was retrained to obtain a better fit quality. The code is available at https://github.com/ LBHB/nems db.

To remove blink artifacts, rapid and transient changes in pupil size were identified (McGinley et al., 2015). The derivative of the pupil trace was taken and bins with derivatives $>6$ SDs from the mean were marked. Blinks were identified within these bins by screening for decreases in pupil size followed by increases. Data during a 6-s period surrounding the blink were removed from the trace and replaced by a linear interpolation of the pupil size immediately before and after the blink.

Pupil size was defined as the length of the minor axis (in pixels) of the fit ellipse. Frame rate of the cameras varied between 10 and 30 frames/s. A timestamp was recorded at the start and end of each trial. Pupil measurements were aligned and interpolated to match the simultaneously recorded neural data. This procedure ensured that the two data streams (video and neural recording) remained synchronized throughout each recording, even with occasional dropped video frames. Pupil data were shifted by $750 \mathrm{~ms}$ relative to spike times to account for the lagged relationship between changes in pupil size and neural activity in auditory cortex (McGinley et al., 2015).

\section{Target-reward association task}

Following recovery from cuff implant surgery, the animals were trained on a tone frequency-reward association using classical conditioning. On each behavioral trial, a pure tone target $(\mathrm{T} 1,1 \mathrm{~s}, 60-\mathrm{dB} S \mathrm{SL})$ was presented at a random position in a sequence of broadband noise distractor sounds [temporally orthogonal ripple combinations (TORCs; Klein et al., 2000); 1-s duration, 1-s interstimulus interval; Fig. 1A]. Frequencies ranging over $0.2-15 \mathrm{kHz}$ were used as targets because these frequencies fall within the hearing range of both humans and ferrets. During initial training, target frequency was changed often to prevent overtraining on a specific frequency. A reward (0.8- to 1.5-ml Ensure) was delivered immediately after target offset. Liquid reward delivery was controlled electronically with a solenoid valve. Licking activity was monitored by a piezo resistor attached to the lick spout. Licks after tone onset but before reward delivery indicated anticipation of the reward and were interpreted as evidence for learning of the reward association. Trial sounds and reward delivery proceeded independently of whether animals responded to the tones or distractors.

When animals learned to associate $\mathrm{T} 1$ with a liquid reward, another tone (T2, frequency two to three octaves away from T1) was presented on randomly interleaved trials but with no reward. In classical conditioning protocols, T1 and T2 are referred to as conditioned stimuli, CS + and CS-, respectively, and the liquid reward is referred to as an unconditioned stimulus (US). Licking is referred to as the unconditioned response (UR). If associative learning occurs, the UR is evoked by the CS+, before the US-. (Menda et al., 2011). In our pilot studies, animals were sometimes able to learn a new target-reward association in $2 \mathrm{~d}$ without VNS pairing. Training on the same association for longer periods made learning of new associations, especially reversals, difficult. Therefore, the frequencies of T1 and T2 were changed every $2 \mathrm{~d}(200-250$ trials/d) in subsequent training sessions (Fig. 1C). This also served to prevent animals from assuming a specific frequency tone was always the CS+ or CS-.

After animals demonstrated an ability to learn new target-reward associations, VNS was introduced during behavior. Two conditions were tested, paired and unpaired VNS-tone. In the paired condition, both T1 and T2 were paired with VNS (1-s duration, $30 \mathrm{~Hz}, 200-\mu$ s biphasic pulses, $0.4-2 \mathrm{~mA}$, starting 100 or $150 \mathrm{~ms}$ before T1/T2 onset). The temporal offset was chosen based on effective protocols reported previously for cortical plasticity following stimulation of vagus nerve (Engineer et al., 2011; Buell et al., 2019) and basal forebrain (Kilgard and Merzenich, 1998). VNS at $100 \mathrm{~ms}$ before T1/T2 onset was used for animals $\mathrm{P}$ and $\mathrm{S}$, and VNS at $150 \mathrm{~ms}$ before T1/ T2 onset was used for animal N. Given the small number of animals, it is not possible to determine whether the timing difference impacted plasticity. The relatively long delay in pupil dilation following VNS (Fig. 2) may reflect the slow dynamics of pupil muscles rather than an extended period of neuromodulatory release (Mathôt, 2018). In the unpaired condition, VNS occurred randomly during the intertrial interval, with a minimum of $500 \mathrm{~ms}$ following T1/T2 offset. Most VNS parameters were taken from Shetake et al., 2012, but the stimulation current was adjusted for each animal. Current was initially set at $0.4 \mathrm{~mA}$, and it was increased in $0.2-\mathrm{mA}$ steps until an effect was observed on learning. The maximal limit of the current was set at $2.0 \mathrm{~mA}$ to avoid causing discomfort during stimulation. The lowest effective current was determined as the level at which the animal showed higher cumulative lick rate for the rewarded tone consistently over $2 \mathrm{~d}$ of training, compared with the unpaired VNS-tone condition. Once this level was determined for each animal, the same current was then used in all subsequent training sessions and neurophysiological recordings (animal P: $1.5 \mathrm{~mA}, \mathrm{~S}: 2.0 \mathrm{~mA}$, and $\mathrm{N}$ : $0.4 \mathrm{~mA}$ ). While animals did not show any overt response to the VNS, they could have perceived it. However, if this was the case, the VNS could not provide an explicit cue for reward, since it was paired with both the rewarded and non-rewarded tones. The impedance of the cuff electrode (5-15 $\mathrm{k} \Omega$ ) was verified during training by converting the voltage required to produce the stimulation current into a resistance value. All data reported in the results were collected after the effective stimulation current was established. 

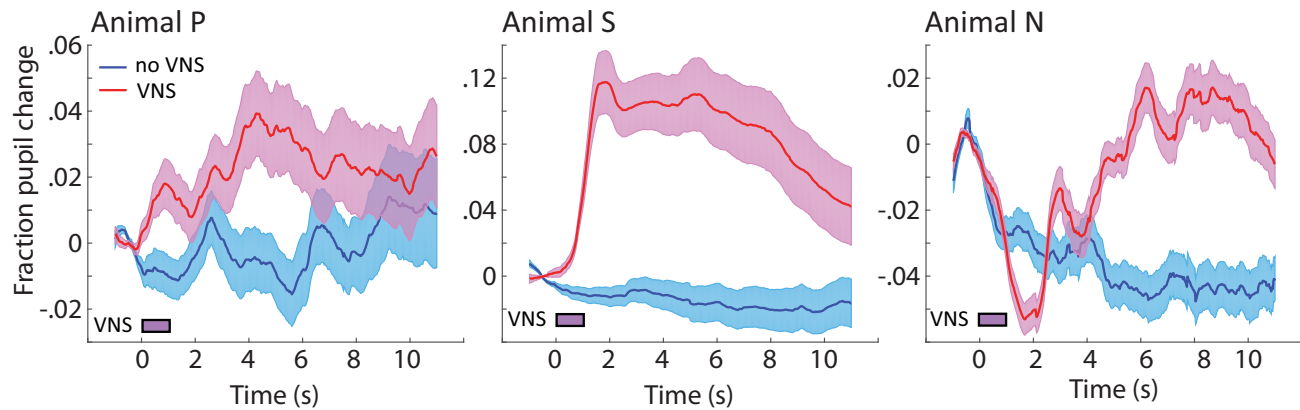

Figure 2. Time course of change in pupil diameter following VNS in silence. Fraction change was computed by aligning each trial to VNS onset and normalizing relative to the 1-s period before VNS. Control (no VNS) trials were interleaved with identical timing but without VNS. Animals $\mathrm{P}, \mathrm{S}, \mathrm{N}$ reached maximal dilation at $\sim 4.5, \sim 6$, and $\sim 7 \mathrm{~s}$, respectively. Pupil dilation was significantly increased during and/or after VNS for all animals (P: $T=2.3, p=0.026$; $\mathrm{S}: T=6.4, p<0.0001 ; \mathrm{N}: T=2.4, p=0.018, t$ test) and reached its maximum within $4.5-7 \mathrm{~s}$. Shading indicates SEM.

To quantify animals' learning to discriminate the rewarded (T1) and non-rewarded (T2) target tones, we calculated response rate difference ( $\Delta R$; Kalafut et al., 2014):

$$
\Delta R=R_{T 1}-R_{T 2} .
$$

$R$ is the probability of licking (yes or no lick for every $10 \mathrm{~T} 1$ or T2 trials) during the 0.95 -s window from $0.2 \mathrm{~s}$ after tone onset to $0.15 \mathrm{~s}$ after tone offset. A value of $\Delta R>0$ indicates that the animal preferentially responds to the rewarded tone, T1. No feedback was given in response to licks.

\section{Neurophysiology}

Both neurophysiological and behavioral data were collected from the same animals. Neurophysiological recording was performed in awake, passively listening animals within one to two weeks after behavior data collection was completed. For neurophysiological recordings, a microcraniotomy was opened over A1. Extracellular neurophysiological activity was recorded using one to two tungsten microelectrodes (FHC) or a 64-channel electrode array (Masmanidis Lab, UCLA; Yang et al., 2020). Both the microelectrodes and array were inserted into A1 with independent motorized microdrives (AlphaOmega EPS). Amplified (AM Systems 3600) and digitized (National Instruments) signals were stored using open-source data acquisition software (Englitz et al., 2013). Recording sites were confirmed as being in $A 1$ based on tonotopy and relatively reliable and simple response properties (Shamma et al., 1993; Atiani et al., 2014). During the recording session, animals were observed and monitored by video camera. Acoustic stimulus presentation was controlled by custom MATLAB software (https://bitbucket.org/lbhb/baphy). Digital acoustic signals were transformed to analog (National Instruments), amplified (Crown), and delivered through a free-field speaker (Manger).

To isolate a spiking unit while positioning tungsten electrodes, a pure-tone or broadband noise probe stimulus was played periodically to search for sound-activated neurons. During recordings using 64-channel array, the probe was inserted into auditory cortex until neural activity was observed across the $1.05-\mathrm{mm}$ span of recorded channels. A series of random, brief pure tones (100-ms duration at $60-\mathrm{dB}$ SPL) was used to obtain the tuning curve and determine the best frequency (BF), i.e., the frequency that evoked the strongest spike rate response.

After characterizing tuning properties at a recording site, two frequencies were selected for probing the effects of VNS on tone-evoked activity. The timing of VNS relative to sound onset was matched to that used during behavior in the same animal. One tone was fixed at BF and other two to three octaves away from BF (off-BF). For the 64channel array, the frequency that evoked the maximum response in most neurons was chosen as the BF. After sorting, when a neuron's tuning curve felt within \pm 0.5 octave of the tone, the tone was considered as matching the BF of the neurons. The same tones were presented to passively listening animals in experimental blocks before, during and after a VNS session. The intertrial interval was $12 \mathrm{~s}$, each tone was presented 20 times per block. In the during-VNS block, VNS was either (1) paired with BF tone; (2) paired with off-BF tone; or (3) unpaired with BF tone (VNS onset $6 \mathrm{~s}$ after tone onset). The electrophysiological amplifier was removed during the VNS session to prevent current leakage. Thus, spiking data were not acquired during the VNS session.

Pupillometry was performed during neurophysiological recordings, as described above. It was also performed during a subset of VNS sessions, when neurophysiological recordings were paused. The analysis of pupillometry during VNS versus plasticity following VNS focused on the subset of 71/110 auditory-tuned neurons for which a complete dataset was collected.

Neurophysiological data were processed offline to identify spike events. For tungsten electrode recordings, the raw signals were bandpass filtered at $300-6000 \mathrm{~Hz}$ and then a principal component analysis (PCA)-based clustering algorithm was applied to spike-threshold events (David et al., 2009). For array recordings, single-units and multiunits were sorted offline using Kilosort (Pachitariu et al., 2016). Neurons were considered isolated single units if the SD of events projected onto the spike waveform template was at least two times the noise floor, corresponding to $>95 \%$ isolation of spikes. Spike interstimulus interval (ISI) was also inspected for high collision rates 
Table 1: Statistical tests reported in the Results according to figure numbers

\begin{tabular}{llll}
\hline & Data structure & Type of test & Power \\
\hline Fig. 2 & Normal & $t$ test & $95 \% \mathrm{Cl}$ \\
Fig. $4 A$ & Normal & $t$ test & $95 \% \mathrm{Cl}$ \\
Fig. $4 B, C$ & Normal & rmANOVA & $95 \% \mathrm{Cl}$ \\
Fig. $4 B, C$ & Non-parametric & Pairwise $t$ test & $95 \% \mathrm{Cl}$ with Bonferroni correction \\
Fig. $7 A-C$ & Non-parametric & Wilcoxon rank-sum test & $95 \% \mathrm{Cl}$ \\
Fig. $7 D, E$ & Non-parametric & Wilcoxon signed-rank test & $95 \% \mathrm{Cl}$ \\
Fig. 8 & Non-parametric & Wilcoxon rank-sum test & $95 \% \mathrm{Cl}$ with Bonferroni correction \\
Fig. $9 A$ & Normal & $t$ test & $95 \% \mathrm{Cl}$ \\
Fig. $9 B$ & Normal (residuals) & Pearson's $R$ & $95 \% \mathrm{Cl}$ \\
\hline
\end{tabular}

$\mathrm{Cl}$, confidence interval

$(<1 \mathrm{~ms}$ ISI), but the template projection criterion was adequate to exclude any units with large numbers of collisions.

\section{Evoked activity analysis}

Spike rate and pupil data were binned at $30-50 \mathrm{~Hz}$. Peristimulus time histogram (PSTH) responses to each tone were calculated by aligning spike activity to tone onset and averaging across repetitions. To obtain evoked activity, the mean spike rate during the 0.5-s silence (baseline spontaneous activity) preceding tone onset was subtracted from the PSTH.

Changes in the PSTH response to the BF tone (pre-VNS vs post-VNS) were measured in each of the three VNS conditions (BF-paired, off BF-paired, BF-unpaired). The PSTH was divided into four epochs: spontaneous activity (0-500 ms before tone onset), onset response (0-60 ms after tone onset), sustained response (60-1000 ms after tone onset) and offset response (0-100 ms after tone offset). Average spontaneous rate was subtracted from the other three responses. VNS-mediated changes in onset and offset responses were weak, and the results focus on changes in the sustained response.

\section{Isolation of pupil-related changes by linear regression}

Based on the observation of pupil dilation following VNS and on reports that pupil size is correlated with neural excitability in A1 (McGinley et al., 2015; Schwartz et al., 2020), we hypothesized that persistent changes in A1 activity could be explained by VNS-mediated modulation of pupil dilation. A control analysis was performed to dissociate changes following VNS that could and could not be explained by changes in pupil size. A linear regression model was fitted using 20 -fold cross validation to predict response changes because of fluctuations in pupil size alone. This response $\left(r_{\text {pupil }}\right)$ was estimated using the recorded neural ( $r$ ) and pupil $(p)$ data.

$$
r_{\text {pupil }}=b_{1} * r+b_{2} * p+b_{3} * r * p+b_{0} .
$$

The weights $b_{i}$ are the regression coefficients for effects of pupil size on baseline and response gain. The residual change in response that persisted after accounting for pupil changes $\left(r_{\text {persist }}\right)$ was obtained by subtracting $r_{\text {pupil }}$ from $r$. After regressing out the effects of pupil, a comparison of $r_{\text {persist }}$ before and after VNS was performed in the same way as the comparison of raw evoked responses before and after VNS described above. The pupil-corrected difference in spike rate was computed as postVNS $r_{\text {persist }}$ minus pre-VNS $r_{\text {persist }}$.

\section{Statistical analysis}

When comparing the difference in means between independent samples from two different conditions, a $t$ test was used to assess significance. For multiple pairwise comparisons of data samples, a repeated measure multivariate ANOVA (rmANOVA) was applied to obtain the statistics. In the analysis of sound-evoked activity in A1 neurons, the change in neural response was assessed with rank-sum test and the reduction or increase in mean response was assessed with sign-rank test. Statistics used in each analysis are detailed in Table 1.

\section{Results}

Three ferrets (animals $\mathrm{P}, \mathrm{S}$, and $\mathrm{N}$ ) were implanted with a cuff electrode for VNS. They were trained to perform the target-reward association task using classical conditioning (Fig. 1). Finally, single-unit neurophysiological data were recorded from the same animals before and after pairing VNS with acoustic stimuli.

\section{Pupil dilation following VNS}

Function of the cuff electrode was validated by measuring changes in heart rate (Nearing et al., 2016; see Materials and Methods) and pupil size following VNS (Bianca and Komisaruk, 2007; Desbeaumes Jodoin et al., 2015; Fig. 2). Pupil size was compared following epochs with and without VNS, in the absence of acoustic stimulation. A significant increase in pupil diameter lasting for several seconds was observed following VNS in all three animals (P: $T=2.3, p=0.026$, peak $4.5 \mathrm{~s}$; $\mathrm{S}$ : $T=6.4$, $p<0.0001$, peak $6 \mathrm{~s}$; $\mathrm{N}: T=2.4, p=0.018$, peak $7 \mathrm{~s}, t$ test). There were some differences in the time course of dilation between animals. The currents of VNS used in this study were calibrated to induce an identifiable learning effect. It is possible that animals differed in effect threshold, relative to their sensitivity to other VNS effects, such as pupil dilation. Alternatively, the variability in time course could reflect between-animal differences in the neural dynamics of coupling between VNS and pupil control centers or in the slow dynamics of pupil muscles. 


\section{A}
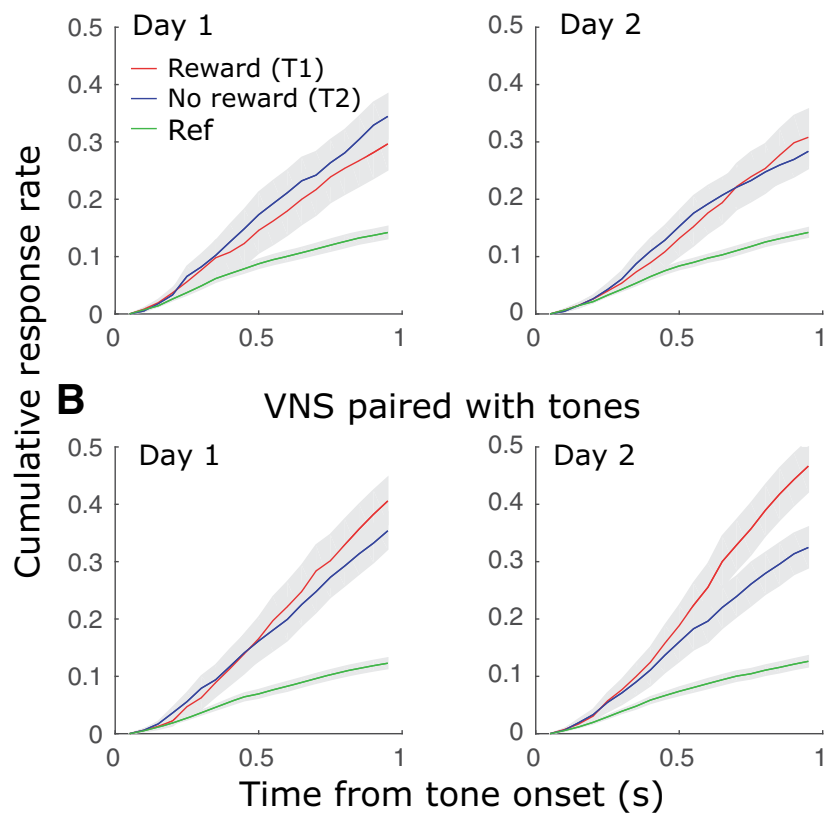

Figure 3. Selective responses to the rewarded tone (T1) over the non-rewarded tone (T2) increased following training. $\boldsymbol{A}$, Average cumulative response rate following sound onset across animals and training conditions (day 1 or day 2 of a tone-reward pairing, 3 animals, 66 reward association conditions total) indicates the probability that the animal licked at least once during presentation of a rewarded tone (T1), non-rewarded tone (T2), or distractor noise (Ref). There was no difference between T1 and T2 on day 1 or day 2 when VNS was unpaired with targets. $\boldsymbol{B}$, When VNS was paired with targets, cumulative response rate to T1 was higher on day 2. Reward was never presented following Ref, and these response rates were lower than for T1 and T2 in both paired and unpaired conditions.

\section{Tone-VNS pairing improved target-reward association learning}

The animals were trained to associate a tone $\mathrm{T} 1$ with a reward (CS+) and T2 with no reward (CS-) by classical conditioning (Fig. 1A). T1 and T2 frequencies and associated reward values were changed pseudo-randomly every $2 \mathrm{~d}$ (200-250 trials/d; Fig. 1C). Paired and unpaired VNS conditions were varied so that transition probabilities were balanced. In addition, transitions between whether the lower-frequency or higher-frequency tone was rewarded were also balanced. Task conditions were changed every $2 \mathrm{~d}$ regardless of performance to prevent overtraining on a single target-reward association. Training was conducted over multiple sessions to cover all the different combinations of transition between VNS pairing and low versus high rewarded frequency. The order of these conditions was pseudo-randomized to control for possible long-term changes in performance. No long-term change in performance was observed across training sessions.

After VNS was paired with both the rewarded (T1) and non-rewarded (T2) tones for $2 \mathrm{~d}$, animals consistently responded more frequently to T1 (Fig. 3B). The selective response to $\mathrm{T} 1$ before reward delivery indicated that the animals learned the new reward association. In contrast, when VNS was unpaired with the tones (occurring between trials), animals did not show consistent evidence of learning the reward categories after the same period (Fig. $3 A$ ). In all cases, average response rate was consistently higher for tones than for the distractor noise, indicating an overall preference for tones over noise, the latter of which was never paired with reward.

To obtain a more detailed characterization of learning across animals and days, performance was measured using the response rate difference $(\Delta R$; Eq. 1$)$, computed by subtracting the response probability to T2 (non-rewarded) from the response probability to $\mathrm{T} 1$ (rewarded; Kalafut et al., 2014). This quantity was measured over blocks of 20 trials within each training session. A positive $\Delta R$ indicated preferential responses to $T 1$, consistent with learning that T1 preceded a reward. A value of 0 indicated equal likelihood of response to T1 or T2, and no learning of the reward association. On training days when VNS was paired with targets, $\Delta R$ was mostly positive and higher than for unpaired VNS-tone sessions. The kernel density estimation of $\Delta R$ of all paired VNS-tone sessions of all animals had a larger positive shoulder compared with that of unpaired VNS-tone sessions (Fig. 4A). Assessment with a $t$ test showed the means of the two VNS conditions were significantly different $(T=-4.5, p<0.05)$.

To summarize the learning data, response rate difference $(\Delta R)$ values for all paired versus unpaired VNS-target conditions were averaged, separately for day 1 versus day 2 and for each animal (Fig. 4B). While one animal showed above-chance performance on day 2 in the unpaired condition (animal $\mathrm{N}$ ), mean $\Delta R$ across all animals was not significantly greater than zero by the end of day 2 $(T=1.41, p=0.16)$. In contrast, the response rate difference was consistently above chance (i.e., $>0$ ) in the paired VNS-tone condition $(T=5.87, p<0.05)$.

The breakdown between training days suggested a consistent pattern of learning reward associations over time, even when average $\Delta R$ was not significantly greater than zero. Multivariate ANOVA (rmANOVA) was used to test for differential effects between days. For all three animals, $\Delta R$ was consistently higher on day 2 compared with day 1 for both conditions $(F=19.546, p<0.05)$. There was no interaction between VNS condition and training day $(F=0.001, p=0.97)$.

Effects of task difficulty on $\Delta R$ were also measured by comparing between blocks in which the relative frequency of T1 and T2 was switched or not switched relative to the previous training sessions (Fig. $4 \mathrm{C}$ ). The frequencies of $\mathrm{T1}$ and T2 changed every $2 \mathrm{~d}$, always maintaining two to three octaves separation and sometimes reversing which tone had higher frequency. An easier "no switch" transition occurred when a new reward association began in which the relative frequency of T1 versus T2 did not change, i.e., T1 had the higher frequency in both the current and previous conditions or had the lower frequency in both conditions. A difficult "switch" condition occurred when the relative frequencies of $\mathrm{T} 1$ and $\mathrm{T} 2$ did change. On both day 1 and day 2 of the paired VNS-tone condition, $\Delta R$ was lower in the more difficult condition. In all 

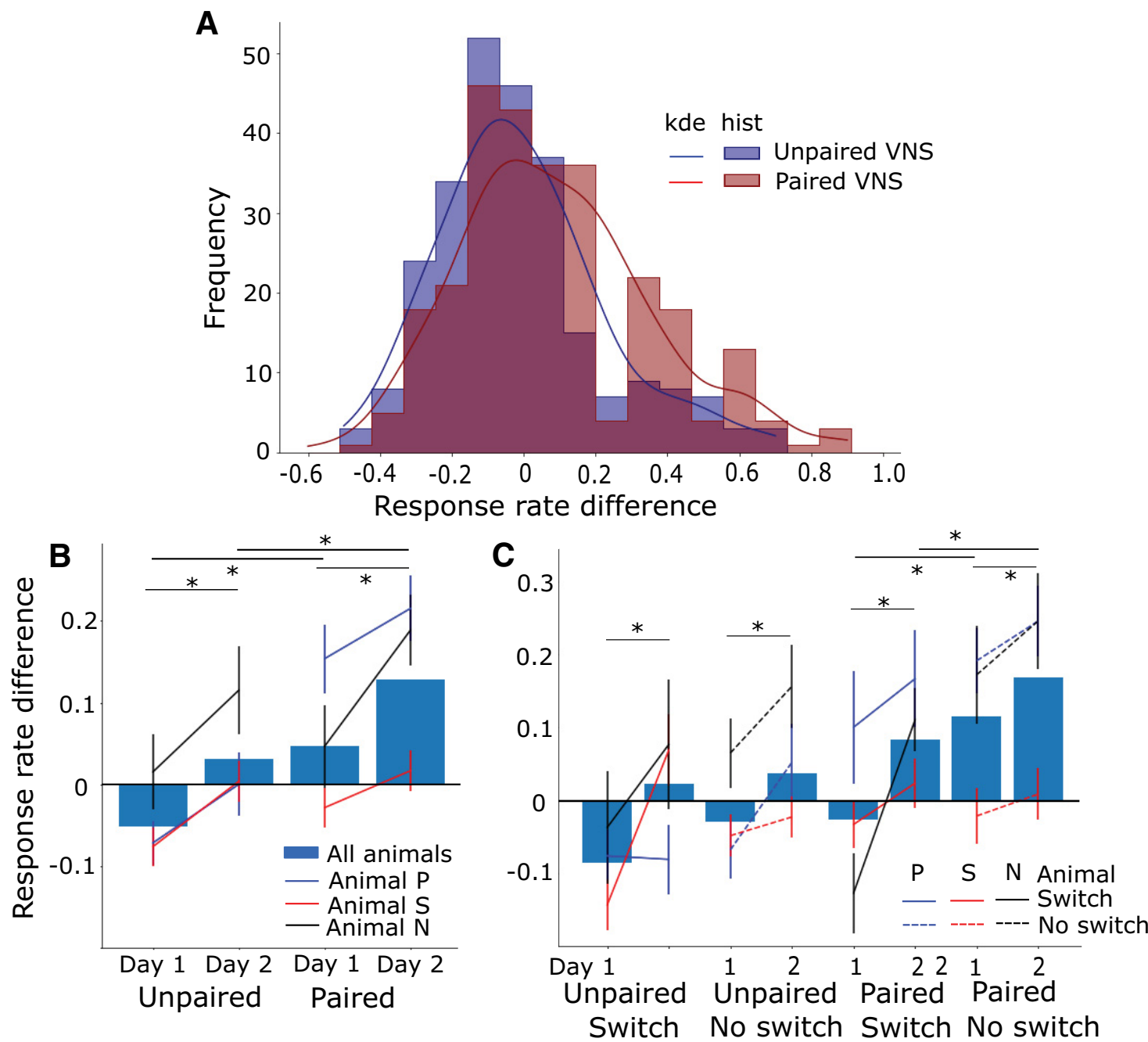

Figure 4. Response rate difference $(\Delta R)$ was larger for paired VNS-tone sessions compared with unpaired sessions. $\boldsymbol{A}$, Histogram (hist) of $\Delta R$ for paired VNS-tone sessions versus unpaired VNS-tone sessions. Lines denote the kernel density estimate (kde) for each histogram. $\boldsymbol{B}$, Mean $\Delta R$ for each day and VNS pairing condition is plotted per animal (lines) and across all animals (bars). Vertical lines indicate SEM. Animals learned to respond preferentially $(\Delta R>0)$ to the rewarded target by day 2 when VNS was paired with tone presentation $\left({ }^{*} p<0.05\right.$ mean difference, rmANOVA). Learning speed (slope between day 1 and day 2 ) was comparable for both paired and unpaired VNS-tone conditions. $\boldsymbol{C}, \Delta R$ subdivided into switch (more difficult, relative T1/T2 frequency reversed from previous session) and no-switch (less difficult, relative frequency not reversed) reward association transitions, plotted as in B. Overall $\Delta R$ was consistently higher when VNS was paired with tone presentation. No significant interaction was observed between task difficulty and day or VNS (rmANOVA, see text for details).

conditions (easy or difficult, paired or unpaired), $\Delta R$ was lower on day 1 compared with day $2(F=18.76, p<0.05$, rmANOVA). The effect of task difficulty did not significantly interact with either VNS condition $(F=1.27, p=$ $0.38)$ or training day $(F=0.03, p=0.88)$.

\section{Reduced sound-evoked activity in A1 neurons following VNS paired with BF tone}

To determine effects of VNS on auditory coding, neural spiking activity was recorded in A1 of awake, passively listening animals before and after VNS. Each pre-VNS and post-VNS session consisted of 20 interleaved presentations of two tones. Across a total of 201 single-units and multiunits in $A 1,110$ had BF near ( $<0.5$ octave) one of the tones. Three tone-VNS pairing conditions were tested: (1) VNS paired with BF tone; (2) VNS paired with off-BF tone; and (3) VNS during intervals between BF tone presentations. This configuration, in which VNS was paired with only a single tone, did not match the paradigm used during behavior, but it permitted measurement of the spectral specificity of plasticity effects. Spiking activity was not recorded during VNS because of the possibly of stimulation current leaking into the recording system. Tone stimulus parameters and VNS current and timing were matched to those used during behavior. PSTH responses to tones pre-VNS and post-VNS were compared. During VNS sessions, a single tone was presented 20 times, with synchronous or asynchronous VNS, as described above. Figure 5 shows the PSTH response of a neuron to the BF 
A

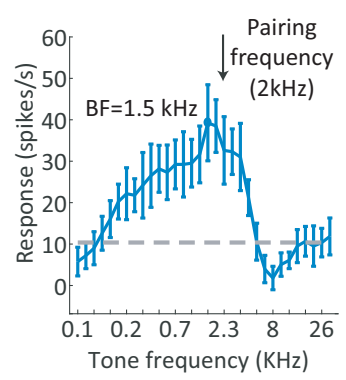

B
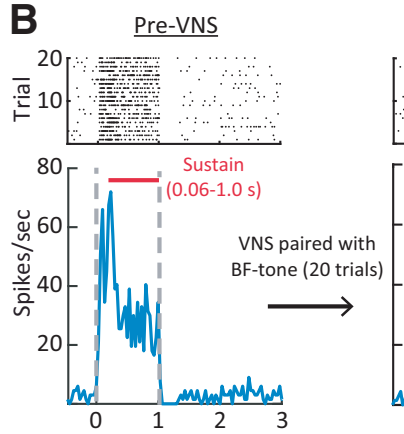

Post-VNS (1)
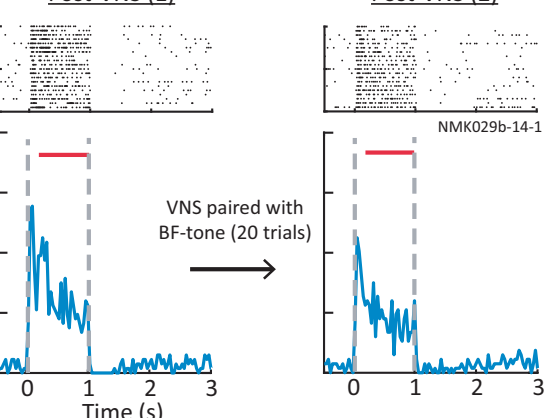

Figure 5. Paired VNS-tone reduced A1 neural responses. A, Example tuning curve of A1 unit measured with brief, random tones. VNS was paired with a $2 \mathrm{kHz}$ tone, close to the best frequency (BF, $1.5 \mathrm{kHz}$ ). $\boldsymbol{B}$, Raster (top) and PSTH (bottom) response of the same unit to BF tone before pairing (left), after one 20-trial session pairing VNS with the BF tone (middle), and after a second 20-trial pairing (right). The PSTH response was reduced following each pairing.

tone before and after VNS paired with the BF tone (toneVNS pairing condition 1).

Since A1 excitability is correlated with pupil size (McGinley et al., 2015; Schwartz et al., 2020) and VNS can increase pupil size by itself (Fig. 2), two possible pathways were considered by which VNS might mediate changes in A1 responses. One pathway is coupled with pupil and induces changes in A1 excitability that reverse after VNSmediated changes in pupil recover. The second pathway promotes plasticity in A1 that persists even after pupil returns to its original size.

To pool VNS-induced pupil fluctuations across experiments, pupil diameter measured during each experiment was normalized by the mean diameter on the first five trials of the pre-VNS session. On average, pupil diameter decreased over the course of each session (Fig. 6A). The large increase in pupil size at the beginning of post-VNS session probably reflected increased arousal after the experimenter entered the anechoic chamber to disconnect the stimulation system. VNS-induced changes in pupil size were smaller and shorter in duration (Fig. 2). Mean firing rate followed a similar pattern to the changes in pupil size, suggesting that changes in arousal, independent of VNS, also affects firing rate (Fig. 6B).

To account for possible effects of arousal on spiking activity, linear regression was performed to measure changes in spontaneous and evoked spike rate that could be accounted for by fluctuations in pupil diameter (Eq. 2; Schwartz et al., 2020). Analysis focused on the 34/201 units with the VNS-paired tone at BF and that underwent significant plasticity following VNS $(p<0.05$, rank-sum test; see Fig. 7A). These changes were classified as pupil effects $\left(r_{\text {pupil }}\right)$, and residual changes, after subtracting pupil effects, were classified as persistent VNS effects $\left(r_{\text {persist }}\right)$. After removing pupil effects, pre-VNS and postVNS spike rates were more stable across trials (Fig. $6 C$ ).

The change in the raw sustained response for each neuron was compared before and after VNS-BF pairing (Fig. 7A). Across the set of $A 1$ neurons with a sustained response, 34/110 (31\%) showed a significant change post-VNS $(p<0.05$, rank-sum test, $11 / 110$ did not produce a sustained response in any condition). For the 34 neurons showing a difference, the mean response was significantly reduced ( $p=0.035$, sign-rank test; Fig. 7E). After regressing out changes that could be attributed to pupil fluctuation, a smaller number of neurons showed significant changes post-VNS $(23 / 110,21 \%$; Fig. $7 B)$, but the decrease in mean response remained $(p=0.006$, signrank test; Fig. 7D). In contrast, the mean residual sustained response was not significantly reduced or increased when VNS was paired with an off-BF tone (Fig. $7 C$ ). There was also not a significant change in the mean response for the unpaired condition, when VNS occurred during the intertrial interval (results summarized in Fig. 7E).

A decrease in response following repeated presentations of the BF tone could reflect adaptation (Ulanovsky et al., 2003). However, the unpaired VNS condition provides a control for auditory adaptation. In this condition, VNS was not overlapped with tone presentation and instead occurred during the intertrial interval, but stimulus timing was the same. The results show no significant change in mean response, indicating that VNS outside of BF presentation has no effect on neural responses. The fact that there is no change in $\mathrm{BF}$ response indicates that there is no measurable adaptation when tones are presented at a rate of once per 12-13 s. Moreover, no significant change in neural responses was observed following VNS paired with off-BF tones (Fig. 7E), providing further evidence against effects of auditory adaptation.

For a subset of recordings from animal $\mathrm{N}$, two sessions of 20 trials pairing VNS with BF tones were repeated. Units with significant changes after either 20 or 40 trials of VNS $(p<0.05$, Bonferroni-corrected rank-sum test) were selected for pre-VNS versus post-VNS comparison. Figure 8 compares pupil-corrected changes in sustained response to the $\mathrm{BF}$ tone. For this small subset, the decrease in response after VNS was not significant. Nonetheless, the mean response showed a trend toward greater reduction as the number of VNS trials increased.

\section{Pupil changes during VNS predict persistent changes post-VNS}

The results above demonstrate relationships between VNS and pupil size (Fig. 2) as well as between VNS and A1 excitability (Fig. 6). Changes in pupil size are 

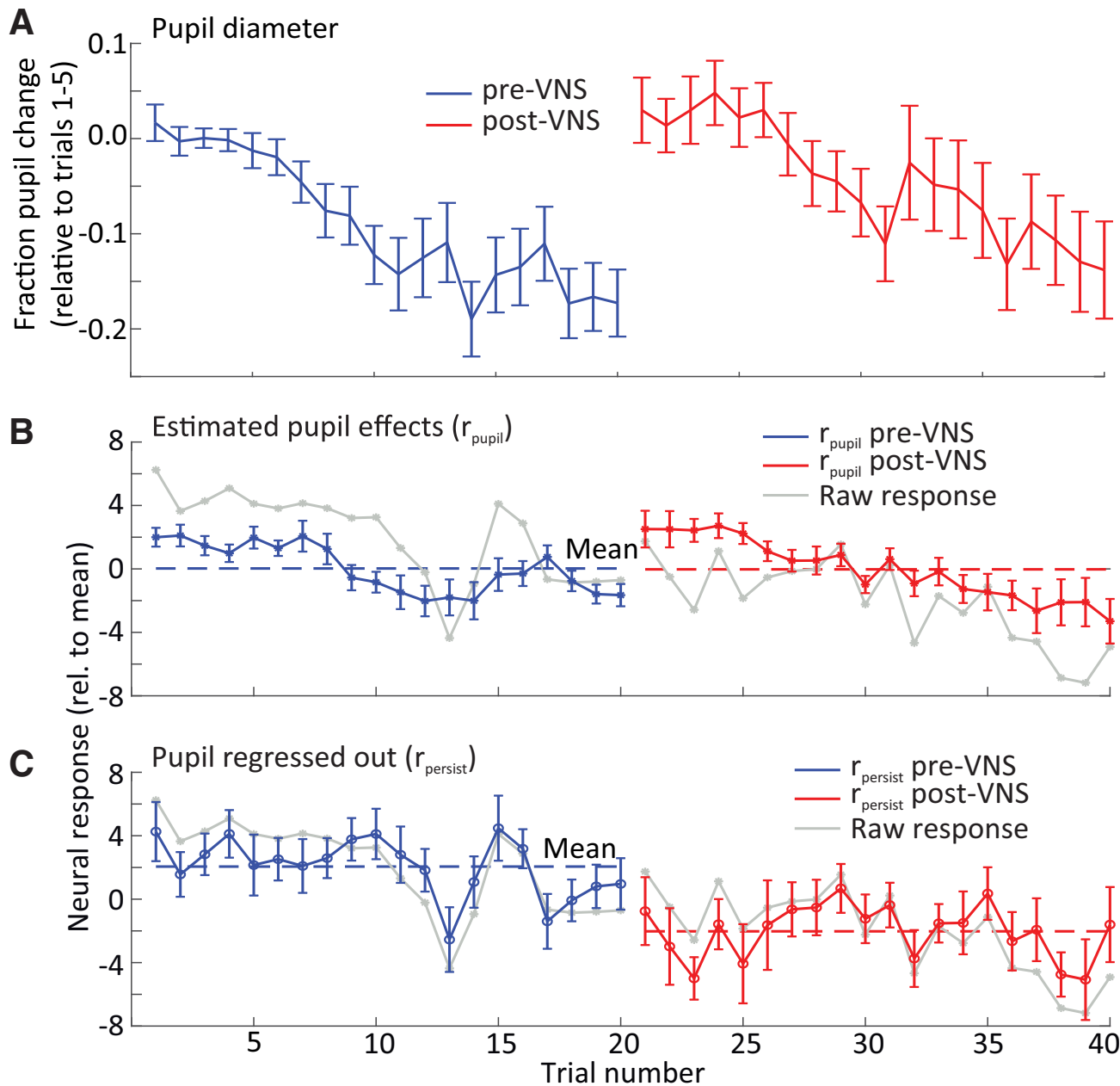

Figure 6. A decrease in mean neural response to best frequency (BF) tones was observed during the post-VNS session. $\boldsymbol{A}$, Fraction change in pupil diameter on each trial pre- and post-pairing of VNS with BF tone presentation, relative to baseline (mean of trials 15 ) and averaged across neurons ( $n=34$ neurons with significant response changes after VNS). Pupil diameter decreased gradually during pre-VNS and post-VNS sessions. $\boldsymbol{B}, \boldsymbol{C}$, Mean change in neural response to BF tones on each trial. The mean change was separated into $(\boldsymbol{B})$ the component that could be explained by pupil fluctuation (as predicted by linear regression) and $(\boldsymbol{C})$ the persistent effect that could not be explained by pupil fluctuation $(n=34)$. The latter component reflects a persistent change in response following VNS. Mean change in the raw response (gray line) is overlaid for comparison. Dashed blue and red lines indicate the mean neural response pre-VNS and post-VNS, respectively. Vertical lines indicate SEM.

associated with neuromodulatory activity (noradrenaline and acetylcholine; Reimer et al., 2016), which in turn is known to mediate cortical plasticity (Dorr and Debonnel, 2006; Engineer et al., 2013). Thus, there is a possibility that persistent changes in spiking activity following VNS could be predicted by changes in pupil size during VNS. For a subset of recordings, pupillometry was measured during VNS-BF tone pairing, allowing analysis of the relationship between pupil size during VNS and response plasticity post-VNS $(n=71)$. Compared with pre-VNS, a larger pupil dilation was observed when VNS was paired with $\mathrm{BF}$ tone presentation $(T=3.2, p=0.0015, t$ test, preVNS versus during VNS; Fig. $9 A$ ), consistent with the VNS-evoked dilation reported above (Fig. 2). Moreover, there was a trend toward larger pupil dilation during VNS when units showed significant persistent changes postVNS $(T=1.7, p=0.086$; compare red and blue lines during VNS in Fig. $9 A, n=19$ modulated, 52 non-modulated units). Across experiments, the mean evoked dilation during paired VNS-BF tone sessions is correlated with the magnitude of the subsequent change in $\mathrm{BF}$ tone response (Pearson's $R=0.32, p=0.0062, n=71$; Fig. 9B, magnitude computed as the absolute value of $r_{\text {persist }}$. These results indicate that changes in pupil size during VNS predict the magnitude of persistent plasticity following VNS. This finding is consistent with the hypothesis that changes in neuromodulatory tone, reflected in pupil size, gate the long-term effects of VNS on sound-evoked activity.

\section{Discussion}

Previous studies have shown that extended periods of pairing VNS with acoustic stimuli (300 times/d for $20 \mathrm{~d}$ ) can induce plasticity in sound coding by $\mathrm{A} 1$ (Engineer et al., 2011, 2015; Borland et al., 2016; Buell et al., 2018) and auditory midbrain (Borland et al., 2019). Building on 
A

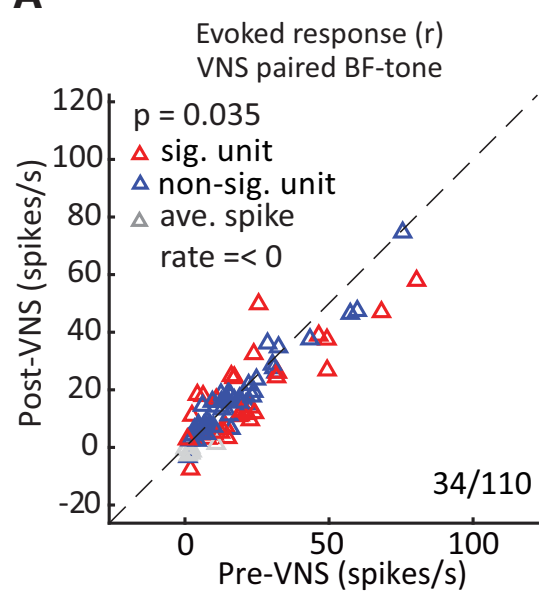

D

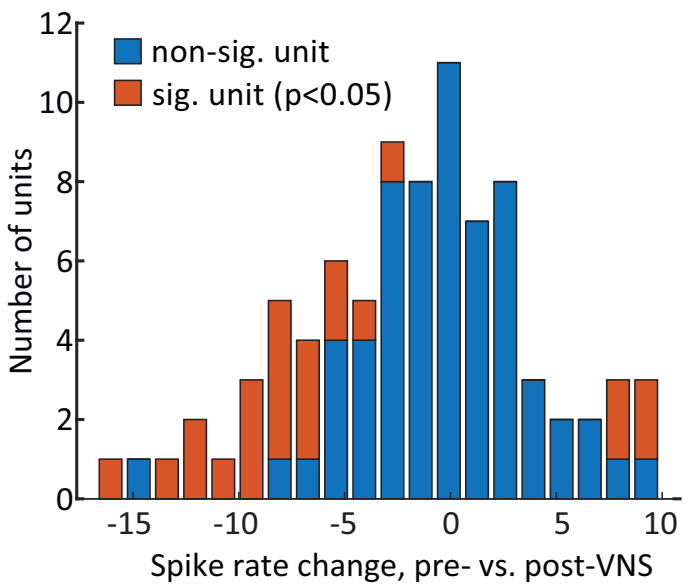

(spikes/s) B
Pupil-corrected response $\left(r_{\text {persist }}\right)$
VNS paired BF-tone

C

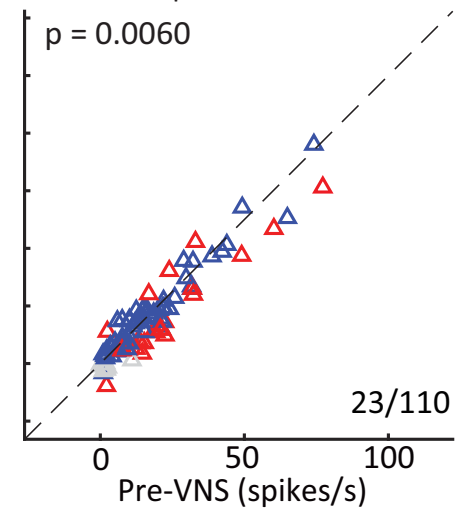

Pupil-corrected response $\left(r_{\text {persist }}\right)$

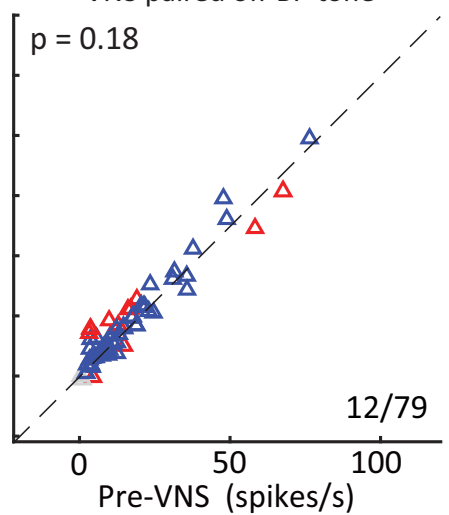

E

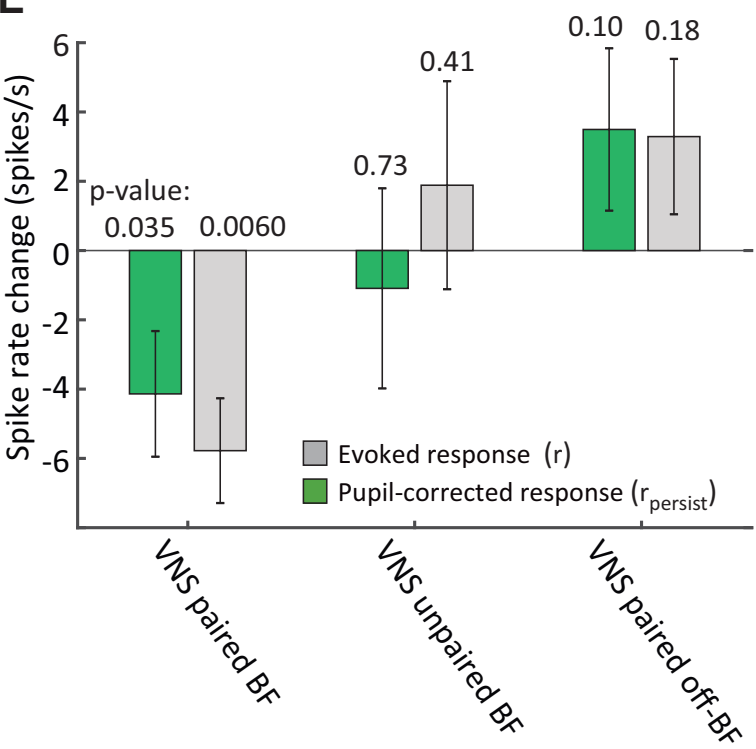

Figure 7. Reduced sound-evoked response in A1 after pairing VNS with best frequency (BF) tone presentation. $\boldsymbol{A}$, Scatter plot compares the sustained BF tone response for each A1 unit prepairing and postpairing of VNS with the BF tone. Red markers indicate units with a significant difference (sig. unit, $p<0.05$, rank-sum test). Numbers (right lower corner) indicate counts of neurons with significant difference. $\boldsymbol{B}$, Scatter plot comparing difference in sustained response after regressing out changes that can be explained by fluctuations in pupil-indexed arousal, plotted as in the left panel. The pupil-corrected response was defined $r_{\text {persist }}=r-r_{\text {pupil }}$, and $r_{\text {pupil }}$ was generated by the model in Equation 2. C. Scatter plot of sustained response before and after pairing VNS with an off-BF tone. As in the middle panel, effects of pupil size on spike rate were removed by linear regression. $\boldsymbol{D}$, Histogram of post-VNS change in spike rate for each unit after removing changes in spike rate explained by pupil fluctuation $\left(r_{\text {persist }}\right)$. Most units with significant changes (red) decreased the response following VNS. Data shown were recorded in the VNS paired with BF tone condition, from panel $\boldsymbol{A}$. $\boldsymbol{E}$, Mean difference in sustained response post-VNS versus pre-VNS, for units with significant changes in auditory responses, under different VNS pairing conditions. Numbers above each bar indicate significance of the mean change (sign-rank test). Vertical lines indicate SEM.

this work, the current study characterized short-term effects of VNS (200 times/d for 1-2 d) on auditory learning and stimulus-specific activity in A1. When VNS was paired with both rewarded and unrewarded tones during classical conditioning, animals responded preferentially to the rewarded tone, indicating learning of its association with the subsequent reward (Menda et al., 2011). Learning was weaker and less consistent in an unpaired VNS condition. Similar enhancements in learning were observed across difficulty conditions. These results demonstrate that short periods of VNS can have significant impact on auditory learning.

In addition to enhanced learning on short timescales, A1 neurons in passively listening animals showed a selective reduction in response after relatively brief pairing of VNS with acoustic stimuli. This reduction persisted even after regressing out changes in spiking that could be explained by fluctuations in the pupil size, which reflect changes in global arousal (McGinley et al., 2015; Schwartz et al., 2020). Pupil dilation is observed following 


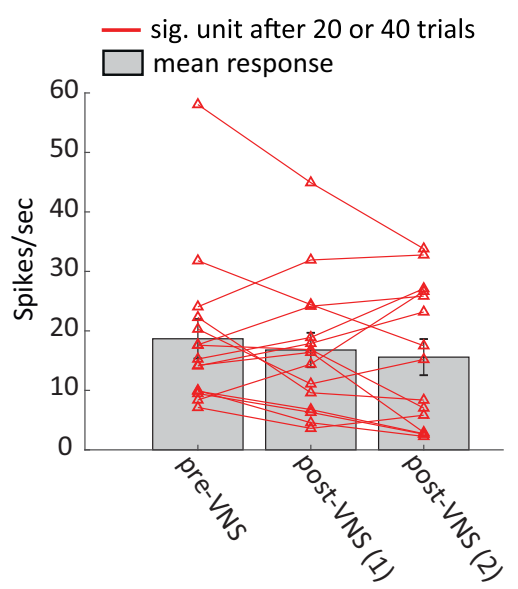

Figure 8. Reduced mean response for neurons with significant changes (sig. unit, $p<0.05$, Bonferroni-corrected rank-sum test) after first or second session of 20 trials pairing VNS with $\mathrm{BF}$ tone. Most neurons with significant changes show a trend toward decreased residual (pupil-corrected) response as the number of paired VNS-tone trials increased from 20 to 40. Vertical lines on bars indicate SEM.

VNS (Bianca and Komisaruk, 2007; Desbeaumes Jodoin et al., 2015) and has been proposed as a readout of VNS efficacy (Mridha et al., 2021). Thus, these results provide evidence that changes in the activity of $A 1$ neurons following VNS-sound pairing are mediated by two pathways (Fig. 10). First, short-term, reversible changes in excitability correlate with changes in pupil-indexed arousal (Schwartz et al., 2020). Second, persistent changes in neural activity, reflecting synaptic plasticity and learning, last longer than changes in pupil size. These effects may not emerge in cortex, as VNS can impact subcortical areas, including the inferior colliculus and thalamus (Borland et al., 2019). Thus, the effects of VNS observed in $A 1$ could be inherited from the ascending auditory pathway. Studies using this same approach in subcortical areas can determine whether either pupil-mediated effects or VNS-mediated effects occur at these earlier processing stages.

These pathways may not be independent, as the longterm effects may be gated by the short-term fluctuations in neuromodulatory activity. Analysis of the relationship between pupil fluctuations during VNS and the persistent changes in neural responses following VNS revealed that they were in fact correlated (Fig. 9). This finding supports the idea that the rapid and reversible effects of VNS, which produce dilation in pupil diameter, positively gate long-term plasticity in A1 (Fig. 10). During neurophysiological recordings, neural responses of post-VNS were recorded within $60 \mathrm{~min}$ following the completion of the VNS protocol. During this period, any dynamics in VNS-related plasticity that could not be explained by dynamics of the pupil were not observed. Based on previous studies using chronic stimulation (Engineer et al., 2011; Borland et al., 2019), VNS can produced long-lasting changes. The changes observed after correcting for pupil effects in this study could be related to this long-term plasticity. Experiments that measure changes over longer time periods post-VNS can confirm this hypothesis.

\section{Impact of VNS on behavioral training and learning}

Although research into the effects of VNS on auditory learning is limited, there is substantial evidence for positive effects of pairing VNS with rehabilitation during recovery from motor disorders (Hays et al., 2014a,b; Khodaparast et al., 2014, 2016). VNS has also been shown to reduce
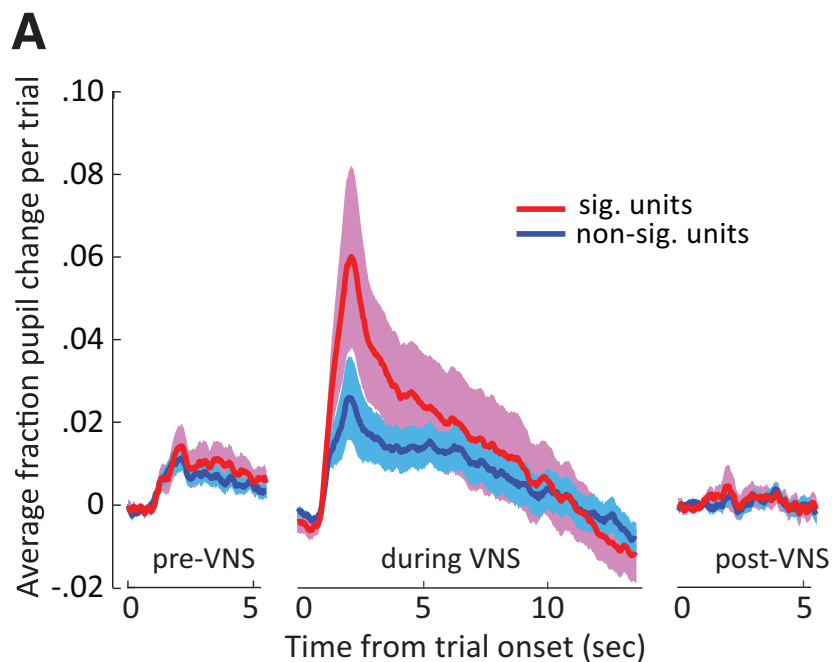

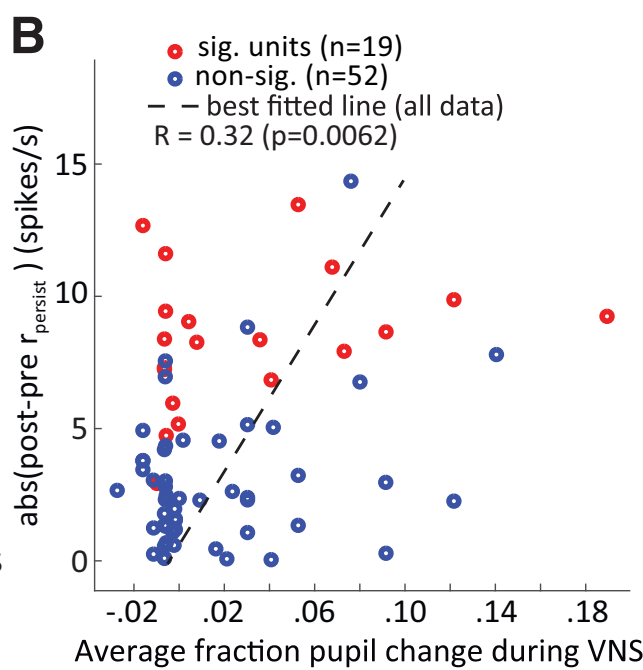

Figure 9. Units with significant changes between pre-VNS and post-VNS sessions were associated with larger changes in pupil size during VNS pairing. $\boldsymbol{A}$, Average change in pupil diameter on each VNS pairing trial, with change measured relative to mean pupil size during the first $0.8 \mathrm{~s}$ of each trial. Data shown are for VNS paired with BF tone presentation. Pupil changes were grouped by sessions in which units underwent persistent changes post-VNS (sig. units, red) or did not (non-sig. units, blue). Changes in pupil size were always large during VNS sessions, but the changes were especially large during sessions that produced persistent changes in neural responses. Shading indicates SEM. B, Scatter plot compares the magnitude of persistent changes in neural response post-VNS against trial-evoked changes in pupil size during the preceding VNS (Pearson's $R=0.32, p=0.0062$ ). 


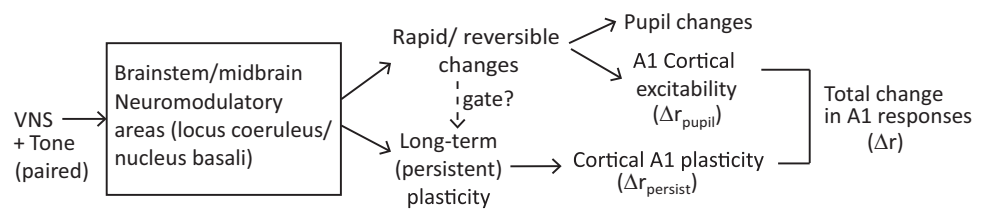

Figure 10. Two possible pathways by which VNS could mediate changes in A1 spiking activity. In the short-term, reversible pathway, VNS evokes changes in pupil size that are correlated with neural excitability. In the long-term, persistent pathway, which may be gated by the rapid and reversible changes, VNS produces long-term plasticity related to learning. The net change in the A1 response is the sum of short-term and long-term effects, $\Delta r=\Delta r_{\text {pupil }}+\Delta r_{\text {persist }}$.

conditioned fear when paired with the conditioned cue during fear extinction in rats (Peña et al., 2013, 2014). Even when extinction training was delayed for two weeks after initial conditioning, animals receiving paired VNS showed significantly less fear response than sham control rats after a single day of extinction training (Peña et al., 2013). This finding is similar to the current results: animals receiving paired VNS-tone during training responded to the rewarded tone more frequently and showed significant and consistent learning effects on the second day of training (Figs. 3B, 4B,C). In contrast, under the unpaired VNS-tone condition, animals did not show consistent learning effects within the same training period. Animals are able to learn similar reward associations without VNS over longer periods (Kuchibhotla et al., 2017). In this study, we limited the duration of training on a single tone-reward association to $2 \mathrm{~d}$ to avoid overtraining on a specific rewarded tone, which can require many training sessions to reverse (Happel et al., 2014).

Previous studies of VNS in auditory learning have involved relatively simple acoustic discrimination or detection (Peña et al., 2013). Similarly, in the current study, rewarded and non-rewarded tones were separated by more than one octave. Thus, the major impact of VNS may have been on learning stimulus-reward associations rather than the discrimination of very similar stimuli. Noble et al. (2019) provide evidence that VNS enhances extinction not only for the stimulus paired with VNS but also for another CS associated with the same fear experience but not paired with VNS. Thus, it remains unclear whether VNS-sound pairing is more beneficial to learning new acoustic categories or learning reward associations with known categories.

\section{Importance of VNS timing and neuromodulator release in plasticity}

Stimulation of vagus nerve triggers the release of neuromodulators from multiple nuclei to drive plasticity, including noradrenergic (LC), cholinergic (NB), and serotonergic (dorsal raphe nucleus) systems (Dorr and Debonnel, 2006; Nichols et al., 2011; Hulsey et al., 2019). A reduction of either noradrenergic or cholinergic signaling prevents VNSdependent effects in the central nervous system, further suggesting that VNS engages these systems (Krahl et al., 1998; Nichols et al., 2011). Furthermore, the importance of precise timing of VNS in the induction of plasticity has also been emphasized in many studies (Engineer et al., 2011; Porter et al., 2012). Neuroplasticity is strongly influenced by the relative timing of stimuli and neuromodulator release. As a result, pairing VNS with a sensory input, for example a movement or an acoustic stimulus, improves recovery from brain injury or tinnitus by enhancing neuroplasticity in a timing-dependent manner (Hays et al., 2013; Khodaparast et al., 2014, 2016; Pruitt et al., 2016). According to the current results, animals learned reward associations faster in the paired VNS-target condition when VNS was synchronized with target tone presentation. This finding is consistent with the hypothesis that precise timing of neuromodulator release is required for the beneficial neuroplasticity associated with auditory learning.

\section{VNS effects on $A 1$}

There is growing evidence of parallels between effects of VNS and direct neuromodulatory stimulation. For instance, acute stimulation of NB causes widespread release of acetylcholine and can enhance the reliability of sensory coding (Goard and Dan, 2009), as well as promote learning and memory (Miasnikov et al., 2008). In their seminal work on auditory plasticity, Kilgard and Merzenich (1998) demonstrated that electrical stimulation of the cholinergic basal forebrain (NB) simultaneous to a sensory stimulus drives plasticity in auditory cortex that mimics plasticity induced by perceptual learning. Furthermore, by pairing more complex stimuli with NB stimulation, selective enhancement has been induced in $\mathrm{A} 1$ for a wide range of acoustic features, including sound level, temporal modulation, and frequency (Kilgard and Merzenich, 1998; Kilgard et al., 2001, 2002; Pandya et al., 2005). Noradrenergic activation may have a similar effect. Repeatedly pairing a tone with LC stimulation also induces selective plasticity for the paired frequency in A1 (Edeline et al., 2011; Glennon et al., 2019). However, NB or LC stimulation is usually performed via highly invasive deep brain stimulation, which could be harmful to the brain and thus has limited therapeutic potential. Because VNS may generate neural plasticity similar to that associated with NB stimulation (Hays et al., 2013), it may provide a safer means of stimulation that bypasses the need for deep brain stimulation.

Since afferent fibers of the vagus nerve innervate cells in the nucleus tractus solitarius, which in turn projects to LC and NB (Engineer et al., 2013; their Fig. 1), electrical stimulation of the vagus nerve should have effects similar to direct stimulation of the NB (Engineer et al., 2011, 2013). Indeed, pairing VNS with a $9 \mathrm{kHz}$ tone caused a $79 \%$ increase in the number of $A 1$ neurons with a characteristic frequency near the paired tone compared with 
naive control rats (Engineer et al., 2013). Moreover, repeatedly pairing VNS with rapid 15 pulse/s tone trains increased the temporal following rate of $A 1$ neurons while pairing VNS with slow 5 pulse/s tone trains decreased the temporal following rate (Shetake et al., 2012; Engineer et al., 2013). These effects can generalize across stimuli. After pairing tone trains at rapid 15 pulse/s with VNS, A1 responses were also increased for unpaired novel speech sounds (Engineer et al., 2017). It has also been demonstrated that VNS modulates synchrony and excitability in the A1 at least in part through the activation of muscarinic acetylcholine receptors (Nichols et al., 2011).

The general observation from studies that perform chronic VNS has been of enhanced firing rate of stimulusspecific neural A1 responses (Engineer et al., 2015; Borland et al., 2019). However, the short-term effects of VNS may be different. Nichols et al. (2011) reported that shorter bouts of VNS increased and decorrelated spontaneous activity of $A 1$ neurons and suppressed entrainment to repeated $6-$ to $8-\mathrm{Hz}$ noise stimulation. This study recorded multi-unit activity in layers $4 / 5$ of anesthetized rat A1 and performed 100 repetitions of VNS (500-ms train of $500-\mu \mathrm{s}$ biphasic pulses at $30 \mathrm{~Hz}$ ) repeated every $10 \mathrm{~s}$ (Nichols et al., 2011). In the current study, both single-unit and multiunit activity in A1 of awake animals were recorded. A total 20 or 40 repetitions of VNS was performed ( $1 \mathrm{~s}$ train of $200-\mu \mathrm{s}$ biphasic pulses at $30 \mathrm{~Hz}$ ) by repeating every $12.5 \mathrm{~s}$. Despite some differences in methodology and parameters used for acute VNS, a suppression of evoked A1 responses after VNS was observed (Figs. 5, 7; similar to the observation by Nichols et al., 2011). While only a relatively small portion of recorded neurons underwent modulation because of VNS, the majority of them were suppressed. It is also important to note that some responses were enhanced and many others did not undergo plasticity after VNS. Within the BF-paired data, we were unable to identify a functional property of units that predicted the sign of plasticity or whether a unit underwent plasticity at all. It is possible that the sign of VNS-related plasticity depends on cell type or layer. A larger dataset or one that identifies cortical layer or cell type may be able to explain the variability in the sign of effects.

The explanation for why short-term VNS leads to suppressed A1 responses while long-term VNS tends to enhance responses remains unclear. Other than differences in the number of pairings between long-term and shortterm VNS, the differences in A1 neural responses could also be attributed to previous pairing of VNS and rewards with sounds before neurophysiological recording. In the previous long-term VNS studies, animals only passively listened to the sounds, and they were never associated with a reward (Engineer et al., 2015; Borland et al., 2019). In addition to opposite effects on evoked activity, changes in spontaneous rate also differ. According to Borland et al. (2019), spontaneous rate was reduced in A1 of rats after long-term VNS-tone pairing, contrasting with the increase reported by Nichols et al. (2011). These differences could reflect a non-monotonic relationship between the number of VNS trials and subsequent plasticity.
Alternatively, slow compensatory processes, such as changes in inhibitory network tone, could alter evoked activity over a longer time following VNS.

The observation of suppression acutely following VNS is also consistent with studies of behavior. Following engagement in some auditory tasks, average evoked activity in auditory cortex is suppressed. The specific pattern of enhancement versus suppression could depend on motor and reward contingencies of the behavior (David et al., 2012) or the balance of excitatory and inhibitory activity in the local circuit (Kuchibhotla et al., 2017). Also possibly relevant, many recordings following chronic VNS have been performed in anesthetized animals, and changes in A1 responses induced by VNS could be masked or affected by anesthesia (Cheung et al., 2001).

\section{Pupil dilation and gating of long-term VNS effects}

Recent work has shown that neuromodulatory activity is correlated with luminance-independent changes in pupil diameter (Murphy et al., 2014; Desbeaumes Jodoin et al., 2015; Joshi et al., 2016). Spontaneous fluctuations in pupil size are correlated with changes in sensory cortical activity (McGinley et al., 2015; Vinck et al., 2015) and track rapid changes in activity of adrenergic and cholinergic axon terminals in cortex (Reimer et al., 2016). Pupil dilation has also been observed following VNS (Bianca and Komisaruk, 2007; Desbeaumes Jodoin et al., 2015) and has been proposed as a biomarker for effective stimulation (Mridha et al., 2021). Because both VNS and changes in pupil diameter are associated with fluctuations in cortical activity, the possibility that acute effects of VNS in A1 could be explained by the global changes in arousal, reflected in pupil dilation, was considered. To control for this possibility, the pupil effects were removed using linear regression. However, significant residual changes in A1 activity were observed (Fig. 7), even after the regression.

When the correlation between pupil size changes during VNS and the persistent changes in A1 activity following VNS was analyzed, a significant correlation was observed (Fig. 9). The association between the size of VNS-evoked changes in pupil and persistent changes in A1 responses suggests that plasticity in A1 is mediated by two pathways. These consist of a short-term, reversible pathway and a long-term, persistent pathway (Fig. 10). In the short-term pathway, changes in pupil size correlate with A1 excitability (Schwartz et al., 2020). In the long-term pathway, which may be gated by activation of the short-term pathway, VNS promotes persistent A1 plasticity that is related to learning improvement. Both short-term and long-term effects can be driven by VNSmediated changes that occur downstream of VNS via neuromodulation.

This study corroborates evidence that acute stimulation of the afferent vagus nerve is associated with enhanced learning of sound-reward categories and with changes in stimulus-specific activity in A1. The relatively rapid effects of VNS on behavior may reflect enhanced learning of sound-reward associations rather than perceptual learning of sounds categories, and future studies may dissociate these effects. The observation that the magnitude of 
post-VNS plasticity depends on the size of evoked pupil dilation during VNS suggests that the effectiveness of VNS depends on the arousal level of the subject. Appropriately timed VNS during training may strengthen learning effects when adults acquire auditory prosthetics or new languages.

\section{References}

Atiani S, David SV, Elgueda D, Locastro M, Radtke-Schuller S, Shamma SA, Fritz JB (2014) Emergent selectivity for task-relevant stimuli in higher-order auditory cortex. Neuron 82:486-499.

Bianca R, Komisaruk BR (2007) Pupil dilatation in response to vagal afferent electrical stimulation is mediated by inhibition of parasympathetic outflow in the rat. Brain Res 1177:29-36.

Borland MS, Vrana WA, Moreno NA, Fogarty EA, Buell EP, Sharma $P$, Engineer CT, Kilgard MP (2016) Cortical map plasticity as a function of vagus nerve stimulation intensity. Brain Stimul 9:117123.

Borland MS, Vrana WA, Moreno NA, Fogarty EA, Buell EP, Vanneste S, Kilgard MP, Engineer CT (2019) Pairing vagus nerve stimulation with tones drives plasticity across the auditory pathway. J Neurophysiol 122:659-671.

Buell EP, Loerwald KW, Engineer CT, Borland MS, Buell JM, Kelly CA, Khan II, Hays SA, Kilgard MP (2018) Cortical map plasticity as a function of vagus nerve stimulation rate. Brain Stimul 11:12181224.

Buell EP, Borland MS, Loerwald KW, Chandler C, Hays SA, Engineer CT, Kilgard MP (2019) Vagus nerve stimulation rate and duration determine whether sensory pairing produces neural plasticity. Neuroscience 406:290-299.

Cheung SW, Nagarajan SS, Bedenbaugh PH, Schreiner CE, Wang X, Wong A (2001) Auditory cortical neuron response differences under isoflurane versus pentobarbital anesthesia. Hear Res 156:115-127.

Clark KB, Krahl SE, Smith DC, Jensen RA (1995) Post-training unilateral vagal stimulation enhances retention performance in the rat. Neurobiol Learn Mem 63:213-216.

Clark KB, Naritoku DK, Smith DC, Browning RA, Jensen RA (1999) Enhanced recognition memory following vagus nerve stimulation in human subjects. Nat Neurosci 2:94-98.

David SV, Mesgarani N, Fritz JB, Shamma SA (2009) Rapid synaptic depression explains nonlinear modulation of spectro-temporal tuning in primary auditory cortex by natural stimuli. J Neurosci 29:3374-3386.

David SV, Fritz JB, Shamma SA (2012) Task reward structure shapes rapid receptive field plasticity in auditory cortex. Proc Natl Acad Sci USA 109:2144-2149.

Desbeaumes Jodoin V, Lespérance P, Nguyen DK, FournierGosselin M-P, Richer F (2015) Effects of vagus nerve stimulation on pupillary function. Int J Psychophysiol 98:455-459.

Dorr AE, Debonnel G (2006) Effect of vagus nerve stimulation on serotonergic and noradrenergic transmission. J Pharmacol Exp Ther 318:890-898.

Edeline JM, Manunta Y, Hennevin E (2011) Induction of selective plasticity in the frequency tuning of auditory cortex and auditory thalamus neurons by locus coeruleus stimulation. Hear Res 274:75-84.

Engineer ND, Riley JR, Seale JD, Vrana WA, Shetake JA, Sudanagunta SP, Borland MS, Kilgard MP (2011) Reversing pathological neural activity using targeted plasticity. Nature 470:101104.

Engineer ND, Møller AR, Kilgard MP (2013) Directing neural plasticity to understand and treat tinnitus. Hear Res 295:58-66.

Engineer CT, Engineer ND, Riley JR, Seale JD, Kilgard MP (2015) Pairing speech sounds with vagus nerve stimulation drives stimulus specific cortical plasticity. Brain Stimul 8:637-644.
Engineer CT, Shetake JA, Engineer ND, Vrana WA, Wolf JT, Kilgard MP (2017) Temporal plasticity in auditory cortex improves neural discrimination of speech sounds. Brain Stimul 10:543-552.

Englitz B, David SV, Sorenson MD, Shamma SA (2013) MANTA-an open-source, high density electrophysiology recording suite for MATLAB. Front Neural Circuits 7:69.

Fritz J, Shamma S, Elhilali M, Klein D (2003) Rapid task-related plasticity of spectrotemporal receptive fields in primary auditory cortex. Nat Neurosci 6:1216-1223.

Glennon E, Carcea I, Martins ARO, Multani J, Shehu I, Svirsky MA, Froemke RC (2019) Locus coeruleus activation accelerates perceptual learning. Brain Res 1709:39-49.

Goard M, Dan Y (2009) Basal forebrain activation enhances cortical coding of natural scenes. Nat Neurosci 12:1444-1449.

Groves DA, Brown VJ (2005) Vagal nerve stimulation: a review of its applications and potential mechanisms that mediate its clinical effects. Neurosci Biobehav Rev 29:493-500.

Happel MFK, Niekisch H, Castiblanco Rivera LL, OhI FW, Deliano M, Frischknecht R (2014) Enhanced cognitive flexibility in reversal learning induced by removal of the extracellular matrix in auditory cortex. Proc Natl Acad Sci USA 111:2800-2805.

Hays SA, Rennaker RL, Kilgard MP (2013) Targeting plasticity with vagus nerve stimulation to treat neurological disease. Prog Brain Res 207:275-299.

Hays SA, Khodaparast N, Hulsey DR, Ruiz A, Sloan AM, Rennaker RL, Kilgard MP (2014a) Vagus nerve stimulation during rehabilitative training improves functional recovery after intracerebral hemorrhage. Stroke 45:3097-3100.

Hays SA, Khodaparast N, Ruiz A, Sloan AM, Hulsey DR, Rennaker RL, Kilgard MP (2014b) The timing and amount of vagus nerve stimulation during rehabilitative training affect poststroke recovery of forelimb strength. Neuroreport 25:676-682.

Hays SA, Ruiz A, Bethea T, Khodaparast N, Carmel JB, Rennaker II RL, Kilgard MP (2016) Vagus nerve stimulation during rehabilitative training enhances recovery of forelimb function after ischemic stroke in aged rats. Neurobiol Aging 43:111-118.

Hulsey DR, Shedd CM, Sarker SF, Kilgard MP, Hays SA (2019) Norepinephrine and serotonin are required for vagus nerve stimulation directed cortical plasticity. Exp Neurol 320:112975.

Johnson RL, Wilson CG (2018) A review of vagus nerve stimulation as a therapeutic intervention. J Inflamm Res 11:203-213.

Joshi S, Li Y, Kalwani RM, Gold JI (2016) Relationships between pupil diameter and neuronal activity in the locus coeruleus, colliculi, and cingulate cortex. Neuron 89:221-234.

Kalafut KL, Freestone DM, Maclnnis MLM, Church RM (2014) Integrating timing and conditioning approaches to study behavior. J Exp Psychol Anim Learn Cogn 40:431-439.

Khodaparast N, Hays SA, Sloan AM, Fayyaz T, Hulsey DR, Rennaker RL, Kilgard MP (2014) Vagus nerve stimulation delivered during motor rehabilitation improves recovery in a rat model of stroke. Neurorehabil Neural Repair 28:698-706.

Khodaparast N, Kilgard MP, Casavant R, Ruiz A, Qureshi I, Ganzer PD, Rennaker RL, Hays SA (2016) Vagus nerve stimulation during rehabilitative training improves forelimb recovery after chronic ischemic stroke in rats. Neurorehabil Neural Repair 30:676-684.

Kilgard MP, Merzenich MM (1998) Cortical map reorganization enabled by nucleus basalis activity. Science 279:1714-1718.

Kilgard MP, Pandya PK, Vazquez J, Gehi A, Schreiner CE, Merzenich MM (2001) Sensory input directs spatial and temporal plasticity in primary auditory cortex. J Neurophysiol 86:326-338.

Kilgard MP, Pandya PK, Engineer ND, Moucha R (2002) Cortical network reorganization guided by sensory input features. Biol Cybern 87:333-343.

Klein DJ, Depireux DA, Simon JZ, Shamma SA (2000) Robust spectrotemporal reverse correlation for the auditory system: optimizing stimulus design. J Comput Neurosci 9:85-111.

Krahl SE, Clark KB, Smith DC, Browning RA (1998) Locus coeruleus lesions suppress the seizure-attenuating effects of vagus nerve stimulation. Epilepsia 39:709-714. 
Kuchibhotla KV, Gill JV, Lindsay GW, Papadoyannis ES, Field RE, Sten TAH, Miller KD, Froemke RC (2017) Parallel processing by cortical inhibition enables context-dependent behavior. Nat Neurosci 20:62-71.

Lu KH, Cao J, Oleson S, Ward MP, Phillips RJ, Powley TL, Liu Z (2018) Vagus nerve stimulation promotes gastric emptying by increasing pyloric opening measured with magnetic resonance imaging. Neurogastroenterol Motil 30:e13380.

Mathôt S (2018) Pupillometry: psychology, physiology, and function. J Cogn 1:16.

McGaugh JL (1989) Involvement of hormonal and neuromodulatory systems in the regulation of memory storage. Annu Rev Neurosci 12:255-287.

McGinley MJ, David SV, McCormick DA (2015) Cortical membrane potential signature of optimal states for sensory signal detection. Neuron 87:179-192.

Menda G, Bar HY, Arthur BJ, Rivlin PK, Wyttenbach RA, Strawderman RL, Hoy RR (2011) Classical conditioning through auditory stimuli in Drosophila: methods and models. J Exp Biol 214:2864-2870.

Meyers EC, Solorzano BR, James J, Ganzer PD, Lai ES, Rennaker RL, Kilgard MP, Hays SA (2018) Vagus nerve stimulation enhances stable plasticity and generalization of stroke recovery. Stroke 49:710-717.

Miasnikov AA, Chen JC, Gross N, Poytress BS, Weinberger NM (2008) Motivationally neutral stimulation of the nucleus basalis induces specific behavioral memory. Neurobiol Learn Mem 90:125137.

Mridha Z, de Gee JW, Shi Y, Alkashgari R, Williams J, Suminski A, Ward MP, Zhang W, McGinley MJ (2021) Graded recruitment of pupil-linked neuromodulation by parametric stimulation of the vagus nerve. Nat Commun 12:1-14.

Murphy PR, O'Connell RG, O'Sullivan M, Robertson IH, Balsters JH (2014) Pupil diameter covaries with BOLD activity in human locus coeruleus. Hum Brain Mapp 35:4140-4154.

Nearing BD, Libbus I, Amurthur B, Kenknight BH, Verrier RL (2016) Acute autonomic engagement assessed by heart rate dynamics during vagus nerve stimulation in patients with heart failure in the ANTHEM-HF trial. J Cardiovasc Electrophysiol 27:1072-1077.

Nichols JA, Nichols AR, Smirnakis SM, Engineer ND, Kilgard MP, Atzori M (2011) Vagus nerve stimulation modulates cortical synchrony and excitability through the activation of muscarinic receptors. Neuroscience 189:207-214.

Noble LJ, Meruva VB, Hays SA, Rennaker RL, Kilgard MP, Mclntyre CK (2019) Vagus nerve stimulation promotes generalization of conditioned fear extinction and reduces anxiety in rats. Brain Stimul 12:9-18.

Pachitariu M, Steinmetz N, Kadir S, Carandini M, Harris Kenneth D (2016) Kilosort: realtime spike-sorting for extracellular electrophysiology with hundreds of channels. bioRxiv e061481.

Pandya PK, Moucha R, Engineer ND, Rathbun DL, Vazquez J, Kilgard MP (2005) Asynchronous inputs alter excitability, spike timing, and topography in primary auditory cortex. Hear Res 203:10-20.

Peña DF, Engineer ND, McIntyre CK (2013) Rapid remission of conditioned fear expression with extinction training paired with vagus nerve stimulation. Biol Psychiatry 73:1071-1077.

Peña DF, Childs JE, Willett S, Vital A, McIntyre CK, Kroener S (2014) Vagus nerve stimulation enhances extinction of conditioned fear and modulates plasticity in the pathway from the ventromedial prefrontal cortex to the amygdala. Front Behav Neurosci 8:327.

Porter BA, Khodaparast N, Fayyaz T, Cheung RJ, Ahmed SS, Vrana WA, Rennaker RL, Kilgard MP (2012) Repeatedly pairing vagus nerve stimulation with a movement reorganizes primary motor cortex. Cereb Cortex 22:2365-2374.

Pruitt DT, Schmid AN, Kim LJ, Abe CM, Trieu JL, Choua C, Hays SA, Kilgard MP, Rennaker RL (2016) Vagus nerve stimulation delivered with motor training enhances recovery of function after traumatic brain injury. J Neurotrauma 33:871-879.

Reimer J, McGinley MJ, Liu Y, Rodenkirch C, Wang Q, McCormick DA, Tolias AS (2016) Pupil fluctuations track rapid changes in adrenergic and cholinergic activity in cortex. Nat Commun 7:13289.

Schwartz ZP, Buran BN, David SV (2020) Pupil-associated states modulate excitability but not stimulus selectivity in primary auditory cortex. J Neurophysiol 123:191-208.

Shamma SA, Fleshman JW, Wiser PR, Versnel H (1993) Organization of response areas in ferret primary auditory cortex. J Neurophysiol 69:367-383.

Shetake JA, Engineer ND, Vrana WA, Wolf JT, Kilgard MP (2012) Pairing tone trains with vagus nerve stimulation induces temporal plasticity in auditory cortex. Exp Neurol 233:342-349.

Ulanovsky N, Las L, Nelken I (2003) Processing of low-probability sounds by cortical neurons. Nat Neurosci 6:391-398.

Vinck M, Batista-Brito R, Knoblich U, Cardin JA (2015) Arousal and locomotion make distinct contributions to cortical activity patterns and visual encoding. Neuron 86:740-754.

Yang L, Lee K, Villagracia J, Masmanidis SC (2020) Open source silicon microprobes for high throughput neural recording. J Neural Eng 17:016036. 